\section{SYNERGISTIC EFFECT OF THE COMBINATION OF ATG- 017, AN ERK1/2 INHIBITOR, AND IMMUNE CHECKPOINT INHIBITOR IN PRECLINICAL CANCER MODELS}

${ }^{2}$ Peng Chen, ${ }^{2}$ Yun Liu, ${ }^{2}$ Min Deng, ${ }^{2}$ Jian Wang, ${ }^{3}$ Dirk Hoenemann, ${ }^{3}$ Kevin Lynch, ${ }^{1}$ Jay Mei, ${ }^{1}$ Bo Shan, 'Bing Hou*. 'Antengene Corporation Co., Ltd, Shanghai, China; 'Shanghai Antengene Corporation Limited, Shang Hai, China; ${ }^{3}$ Antengene Pty Ltd, Melbourne, Australia

Background The RAS/MAPK pathway has emerged as a critical pathway for therapeutic targeting in a spectrum of solid tumor and hematological malignances. Inhibitors targeting MAPK pathway targets, such as RAS, BRAF, or MEK have been approved for the treatment of cancer either as monotherapy or in combination. However, there has been no approved drug targeting ERK1/2, the terminal kinases in the Ras-Raf-MEK-ERK signal transduction cascade. The combination of ERK1/2 inhibitor and checkpoint inhibitors as therapeutic strategy has not been explored in clinic. ATG-017 is an oral, potent, and highly selective inhibitor of ERK1/2, which is under Phase 1 clinical investigation. ATG-012 is a novel inhibitor of KRAS G12C. In this study, we tested the antitumor effect induced by the combination of ATG-017, or ATG-012, and an anti-PDL1 antibody in preclinical cancer models.

Methods We assessed the anti-cancer effects of ERK1/2, KRAS G12C and PD1/PDL1 inhibition as monotherapy, and as combinations. Anti-PDL1 antibody (atezolizumab), the combination of ATG-012 or ATG-017 and atezolizumab, and the triple combination of atezolizumab, ATG-012 and ATG-017 were tested in a $\mathrm{PD}(\mathrm{L}) 1$ blockade insensitive syngeneic lung cancer model,LL/2. The ATG-017-atezolizumab combination was also evaluated in a KRAS G13C-mutant,PD(L)1 blockade insensitive lymphoma model, EL4. To further investigate if ERK1/2 inhibition could enhance the efficacy of atezolizumab in a MAPK aberration-independent manner, the ATG-017-atezolizumab combination was tested in a MAPK wild type lymphoma model, A20. As well as assessment of impact on tumor growth, the impact of the drugs on tumor infiltrating leukocytes were analyzed by flow cytometry.

Results The combination of ERK1/2 inhibition and an antiPDL1 antibody showed enhanced efficacy in mouse syngeneic tumor models. In the EL4 model, neither ATG-017 nor atezolizumab showed single agent activity, while the combination showed significant tumor growth inhibition (TGI=22\%) on day 9. No body weight loss was observed. The percentage of infiltrating CD8 $+\mathrm{T}$ cells, NK cells and CD8:CD4 ratio were found increased in the combination group. The mean percentage of $\mathrm{CD} 8+\mathrm{T}$ cells among CD45+ cells increased from 4.17\% (IgG + Vehicle control), 3.81\% (ATG-017), 3.23\% (atezolizumab), to $12.92 \%$ in the combination group. The CD8: CD4 ratio were 0.25 (IgG+Vehicle), 0.32(ATG-017), 0.17 (atezolizumab) and 0.67 (combination), respectively (Figure 1). More data from LL/2 and A20 model are being generated.

Conclusions Synergism has been observed for the combination of checkpoint inhibition and ERK1/2 inhibition in vivo, suggesting promising therapeutic strategies for cancer patients that warrants further clinical investigation.

Ethics Approval The protocol and any amendment(s) or procedures involving the care and use of animals in this study were reviewed and approved by the Institutional Animal Care and Use Committee (IACUC) of CrownBio prior to execution. During the study, the care and use of animals were conducted in accordance with the regulations of the Association for Assessment and Accreditation of Laboratory Animal Care
(AAALAC).All studies were conducted following an approved IACUC protocol. AUP NO.: AN-2004-09-309
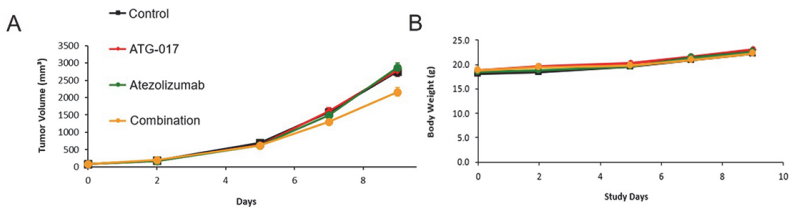

C
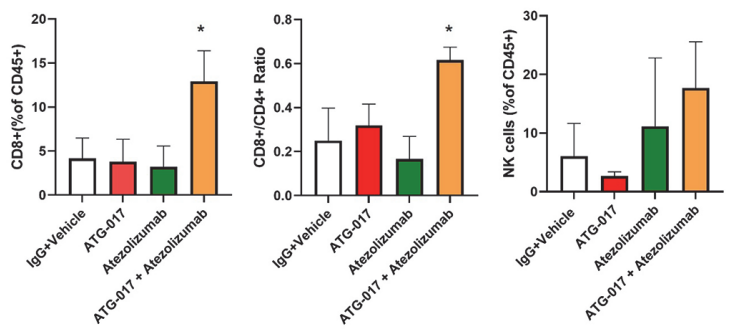

Abstract 608 Figure 1 ATG-017-Atezolizumab combination showed in vivo synergism

(A) Tumor growth curve and (B) mean body weight of EL4 syngeneic model; $\mathrm{N}=8$ for each treatment group. (C) ATG-017-Atezolizumab combination increased tumor infiltrating CD8+T cells (left), CD8+/CD4+ ratio (middle), and tumor infiltrating NK cells (right). The TILs were isolated from tumor samples shown in (A). $\mathrm{N}=3$ for each group.

http://dx.doi.org/10.1136/jitc-2021-SITC2021.608 